Port Acadie

Revue interdisciplinaire en études acadiennes

An Interdisciplinary Review in Acadian Studies

\title{
Chacal, mon frère ou le roman de la frérocité
}

\section{Robert Viau}

Numéro 27, printemps 2015

URI : https://id.erudit.org/iderudit/1038350ar

DOI : https://doi.org/10.7202/1038350ar

Aller au sommaire du numéro

Éditeur(s)

Université Sainte-Anne

ISSN

1498-7651 (imprimé)

1916-7334 (numérique)

Découvrir la revue

Citer cette note

Viau, R. (2015). Chacal, mon frère ou le roman de la frérocité. Port Acadie, (27), 119-130. https://doi.org/10.7202/1038350ar d'utilisation que vous pouvez consulter en ligne.

https://apropos.erudit.org/fr/usagers/politique-dutilisation/ 
NOTE CRITIQUE

\section{Chacal, mon frère ou le roman de la frérocité}

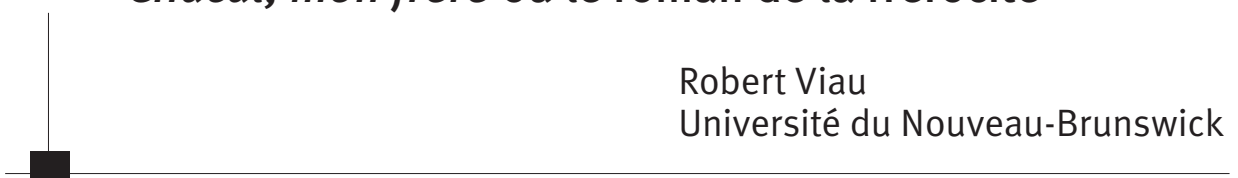

«Suis-je le gardien de mon frère? » (Gn $4: 9)$

Née à Edmundston, Gracia Couturier est auteure, metteuse en scène et comédienne. Son œuvre peut facilement se diviser en fonction des dates et des genres littéraires qu'elle a pratiqués. Ainsi, elle a publié quatre pièces de théâtre de 1986 à 1989, ensuite deux romans en 1997 et en 1999, puis une série d'œuvres pour la jeunesse en 2002-2003. En 2010, elle revient au roman et publie Chacal, mon frère ${ }^{1}$, qui connaît un succès public et critique. L'auteure remporte le Prix des lecteurs Radio-Canada 2011 et le Prix littéraire France-Acadie 2012². Publié en 2010 dans la collection « Voix narratives » des Éditions David (premier tirage épuisé), le roman a ensuite été publié dans le nouveau format poche des Éditions David en 2012.

Chacal, mon frère présente une intrigue à rebondissements, aux éléments abondants et complexes, mais qui développe surtout le thème des frères ennemis ou de la haine fratricide, la «frérocité " ${ }^{3}$. L’irrépressible envie de nuire à son frère ronge un des personnages. Certes, des frères, lorsqu'ils sont jeunes, se disputent avec acharnement l'attention des parents et l'exclusivité de leur amour, et ils peuvent se sentir spoliés par des actions de l'autre, mais cette dynamique relationnelle peut aussi se prolonger très souvent à l'âge adulte et s'exprimer de manière très violente. C'est ce thème de la jalousie fraternelle, sentiment vieux comme

1. Gracia Couturier, Chacal, mon frère, Ottawa, Éditions David, 2012, 309 p. Nous indiquerons entre parenthèses les citations tirées de cet ouvrage par la lettre $C$ suivie de la page.

2. Pour le Prix des lecteurs, voir, sur Internet, le site : http://www.radio-canada.ca/ regions/prixdeslecteurs/archives/2011/prix/. Pour le Prix France-Acadie, voir, sur Internet, le site : http://www.snacadie.org/index.php/nos-dossiers/prix-e t-rompenses-leftmenu-216?id=84: prix-france-acadie \&catid $=73$

3. La «frérocité » est un terme qui a été proposé par José Attal dans «Frère Semblant», Revue du Littoral, $\mathrm{n}^{\circ}$ 30, octobre 1990, p. 33. 
le monde, qu'explore Gracia Couturier, dans un contexte acadien. Jusqu'à quelles limites peut-on laisser ces jalousies s'exprimer? L'extrême violence des sentiments peut-elle mener au crime fratricide? La transgression des interdits ne permet-elle pas au frère spolié - ou se croyant tel - de s'accomplir, d'affirmer son existence? Le Mal, dans toute sa puissance, peut-il alors devenir positif, en ce sens qu'il accroît l'intensité de la vie, permet d'aller « le plus loin possible "? La haine du frère est un thème romanesque aux ramifications troublantes, qu'exploite Couturier dans son plus récent roman et qui soulève plus de questions qu'il n'apporte de réponses.

\section{La rivalité initiale}

Dans Chacal, mon frère, Gracia Couturier raconte la relation tourmentée et la rivalité inquiétante entre deux frères. L'aîné, Bruno Bellefleur, est un être incomplet, hargneux et vindicatif, qui n'arrive pas à se libérer du passé et de ses hantises. Figure morbide, il est présenté, dès les premières pages du roman, reclus dans le silence de la maison paternelle, assis dans la berceuse dans laquelle sa mère le berçait, les jambes recroquevillées, le menton appuyé sur les genoux, les yeux hagards. Replié sur lui-même dans une attitude fœtale, il regarde la télévision, qui diffuse en boucle l'image des tours du World Trade Center qui n'en finissent plus de s'effondrer. L'attentat terroriste renvoie à son propre désir de destruction, aveugle et sans pitié.

L'agitation tragique, les gens qui se sauvent, les décombres qui suivent en débandade rappellent aussi à Bruno la débâcle du printemps, quand la rivière déchaînée emporte tout sur son passage, et elle lui rappelle par ce fait même sa propre naissance. Car Bruno est un être de fureur, né un jour de violence. La colère bouillonne en lui. Sa naissance en avril est liée à la rivière en crue qui a presque fait chavirer le canot dans lequel sa mère était transportée chez la sage-femme, de sorte que "l'histoire de cet enfant qui venait de naître avait pris racine dans la tourmente » $(C, 23)$. La rivière perfide, violente, demeure sa véritable source d'inspiration : " $m a$ mère, ma rivière, la berceuse de mes jours » $(C, 30)$.

Quelques années après sa naissance, Bruno a été déstabilisé par la naissance d'un frère. Cet événement saccage l'univers de l'empremier, de paradis terrestre qu'il s'était construit, car ce frère cadet lui ravit l'amour indivisible que lui vouait sa mère. Bruno voulait cette mère pour lui tout seul, sans partage, sans réserve. Maintenant, il l'observe qui s'occupe de cet être qu'il déteste. Ce frère, Étienne, est né un doux soir de juin, parmi l'odeur des lilas qui entrait dans la maison. Sa naissance, à la différence de celle de Bruno, a été douce, sereine, tranquille. La mission que se donne Bruno sera de faire disparaître cet être si différent de lui, ce 
qu'il entreprend dès le matin de la naissance d'Étienne, lorsqu'il égrène symboliquement des lilas et les regarde disparaître dans le courant de la rivière. Toutefois pour véritablement réussir à chasser l'autre, il faudra faire en sorte que "le soleil se lève parfois / sur la rive ouest / de la rivière » $(C, 12)$, c'est-à-dire qu'il aille contre l'ordre naturel des choses et que le frère assassine le frère.

D’une certaine façon, la naissance d’Étienne a marqué le début des malheurs de la famille Bellefleur : Bruno a régressé; Irène, sa mère, a cessé, pendant longtemps, de peindre (sauf pour un tableau sur le mur de la chambre d'Étienne); un cancer ovarien s'est déclaré et elle est devenue stérile en raison de la radiation des traitements subis. Irène prend alors conscience que "son semblant de vie idéale [est] en train de devenir un carcan qui l'étouff[e] » $(C, 32)$ et elle s'absente de la famille pendant des mois pour voyager, se ressourcer, visiter des galeries d'art, parler avec des artistes. C'est pour ces raisons - et d'autres qui relèvent de ses fantasmes - que Bruno décide que le «petit [devra] pa[yer] pour son crime » $(C, 30)$, c'est-à-dire celui d'être né et de s'être placé entre Bruno et sa mère. Pour Bruno, l'enfer est un autre, son frère. En fait, il ne s'agit pas uniquement d'Étienne, mais de tout frère qui oserait s'interposer. Irène avait fait une fausse couche entre Étienne et Bruno et, dans l'esprit de ce dernier, ses vœux se sont déjà réalisés une première fois, de sorte qu'il ne tient qu'à lui que la mort intervienne de nouveau et qu'Étienne disparaisse à son tour.

Être silencieux et énigmatique, Bruno vit dans une bulle de ressentiment. Il s'enferme dans le cocon de la maison paternelle, il est le seul qui puisse verrouiller la porte de sa chambre et il voyage dans le cyberespace, loin des réalités qui l'importunent. La faute de Bruno est de négliger son frère, de l'éviter en s'enfermant dans sa chambre, de ne pas le regarder face à face, puisque c'est justement ce face-à-face, ce visage de l'autre, dirait Lévinas, "qui nous interdit de tuer ${ }^{4}$. Ce refus de regarder l'autre exacerbe le désir d'homicide et le rend possible. L'acte que veut commettre Bruno s'inscrit dans une thématique ancienne. La rivalité entre Bruno et Étienne est le fond même de l'histoire humaine, car ce récit reprend le symbole du sang versé par l'humanité depuis l'époque de Caïn et Abel.

\section{Les frères ennemis}

Dans la Bible, texte fondateur et source de structures archétypales, il est écrit que Caïn a tué son frère Abel par jalousie, parce que Dieu a préféré ses offrandes aux siennes. Dans ce récit de la Genèse, le mot «frère »

4. Emmanuel Lévinas, Éthique et Infini, Paris, Arthème Fayard, p. 97. 
surgit pour la première fois, mais ce texte décrit aussi l'apparition de la Mort, puisque Caïn a commis le premier meurtre de l'humanité. Quand l'homme se trouve face à son frère pour la première fois, ce n'est pas l'amitié ou l'amour qui est décrit, mais la haine qui éclate dans le meurtre.

De nombreux autres récits mythiques racontent un fratricide. Selon la mythologie romaine, Romulus a tué son frère Rémus qui a transgressé le sillon sacré marquant les limites de sa future ville de Rome. Chez les Égyptiens, Seth, jaloux de son frère, complote et tend un piège à Osiris, qu'il assassine en le noyant dans le Nil et en procédant ensuite à son dépeçage. Chez les Grecs, Polynice et Étéocle, les deux fils d'CFdipe, s'entretuent au cours d'un combat pour la possession du royaume de Thèbes. Comme l'explique Étéocle, dans La Thébaïde de Racine, cette haine date du jour de leur naissance :

Nous avons l'un et l'autre une haine obstinée : [...]

Elle est née avec nous; et sa noire fureur

Aussitôt que la vie entra dans notre cœur.

Nous étions ennemis dès la plus tendre enfance 5

Être frères n'implique pas de s'aimer. Tout être humain est habité de pulsions de haine qui peuvent concerner sa fratrie. Les liens de sang ne garantissent pas une entente et une affection évidentes, quel que soit l'âge des frères. À ce propos, saint Augustin remarque :
Ainsi la faiblesse du corps est innocente chez l'enfant, mais non pas son âme. J'ai vu et observé un petit enfant jaloux : il ne parlait pas encore et il regardait, tout pâle et l'œil mauvais, son frère de lait. Qui ignore le fait? Les mères et les nourrices prétendent conjurer cette envie par je ne sais quels charmes. Dira-t-on que c'est innocence, lorsque la source de lait coule si abondamment, de ne point admettre au partage un frère dénué de tout et qui ne peut soutenir sa vie que par cet aliment? On souffre ces passions avec indulgence, non qu'elles ne comptent pas et soient sans importance, mais parce qu'on croit qu'elles passeront avec l'âge. ${ }^{6}$

Bruno a pour but d'éliminer un rival qui l'empêche d'être unique et de jouir de l'amour sans partage de sa mère. Toutefois, dans le cas de Bruno, il ne s'agit pas d'un " petit enfant jaloux ». La jalousie ne passe pas avec l'âge, s'enracine profondément dans sa psyché, renvoie à cet impossible

5. Jean Racine, La Thébaïde - Théâtre complet 1, Paris, Garnier-Flammarion, 1964, Acte IV, Scène première, p. 70.

6. Saint Augustin, Les Confessions, Paris, Garnier-Flammarion, 1964, I. 1, ch. 7, p. 22. 
de la fraternité, à des affrontements et à des violences de plus en plus cruelles. Cette situation, "qui dure depuis longtemps et que tout le monde tente de dissimuler derrière une apparente normalité » $(C, 45)$, ne cesse de s'envenimer.

Bruno agit en fonction de ce désir effréné de posséder ce que possède l'autre et de faire ce que fait l'autre. D'où le cercle vicieux de la concurrence, de ce besoin de surpasser son frère, sinon de détruire ce qu'il entreprend. Bruno renverse du vin, ou du sang comme il le croit dans son délire, sur la robe de Lorraine, l'amie d'Étienne. Il raconte son rêve d'épouser cette fille qui porterait " une robe blanche sur la rivière " $(C, 41)$, la robe de mariée de sa mère. Si Étienne prend la parole au nom de son père pour rendre un dernier hommage au curé Brisebois, Bruno n'hésite pas à uriner dans la fosse du curé. Si Étienne a une aventure avec Judith, une amie d'enfance, Bruno avertit Lorraine et brise la patte du chien de Judith. Il envoie des courriels ou téléphone aux deux femmes pour brouiller la situation. Étienne doit prendre la direction de la scierie, alors Bruno provoque un incendie qui détruit une partie de celle-ci. Lors de la réouverture de la scierie, rebaptisée Bellefleur et fils, tous signent la première planche, mais Bruno profite d'un moment d'inattention pour rayer le nom d'Étienne. Une nuit, il tente d'asphyxier son frère à l'aide d'un oreiller. De plus en plus laissé de côté, il se venge en sabotant les freins de la voiture de ses parents, ce qui provoque leur mort, puis il vandalise les tableaux de sa mère.

Qu'il ait les mains « souillées de noir » $(C, 161)$ de l'huile des freins de la voiture, "souillées de peinture rouge » $(C, 172)$ des tableaux vandalisés ou tendues au-dessus de l'ordinateur, Bruno manipule les gens en toute impunité. Il est le génie maléfique qui a le pouvoir de faire hésiter, de faire douter, de tuer. Plus que les autres êtres dits " normaux », il possède cette finesse et habileté sans scrupule qui est appelée "rouerie », ainsi que l'intelligence et les ruses permettant d'accomplir toutes choses. Ainsi, il participe aux recherches et enquêtes et les gens ont souvent recours à lui pour régler des problèmes, alors que, bien souvent, c'est lui-même qui en est la cause. De nature fondamentalement négative et traître, sa jalousie l'amène à causer la mort de ses parents et à planifier celle de son frère, sans se soucier des répercussions de ses actes.

\section{Croître grâce à l'autre?}

D'un point de vue psychologique, comment comprendre un tel être? Pour Bruno, Étienne est un faux semblable qui présente une image corporelle familière. Mais dans cette image qu'il présente, Bruno prend conscience que ce n'est pas lui qui a l'objet du désir, c'est l'autre qui s'en satisfait, d'où la nécessité du meurtre du frère, de celui qui s'empare de 
tout - de l'amour de la mère et de l'héritage du père. Bruno veut tuer Étienne, "le préféré », et cet acte meurtrier devient obligatoire dès lors qu'une relation de concurrence s'instaure. Bruno est un personnage complexe, dont les sentiments sont à l'origine de ses actes. Avec le rejet - ou du moins ce qu'il perçoit comme tel - viennent la colère et le désir de vengeance. Bruno se donne pour but d'éliminer son rival, qui l'empêche d'être unique et d'avoir la possession sans partage de l'amour maternel.

Certes, il pourrait s'agir, du point de vue psychologique, d'un frère qui cherche à se distinguer, qui ne s'est pas encore délimité de façon tranchée de l'autre, qui se trouve comme dans un état d'indistinction originaire où le Moi se cherche. La fonction sujet d'un enfant ne peut se mettre en place que par la présence de l'autre qui aide à s'identifier soi-même, comme on s'identifie dans le miroir. L'enfant a besoin de l'autre pour se constituer. Ce frère, qui lui est à la fois opposé et complémentaire, peut lui permettre de s'identifier, l'aider à former une personnalité plus sereine.

Toutefois, dans le roman de Couturier, il n'y a pas de métamorphose, d'évolution des relations entre Bruno et Étienne, d'état de conscience à atteindre. D’une certaine façon, Bruno a raté son stade du miroir, cette étape du développement qui lui permet d'accéder à son identité et, par voie de conséquence, à la notion de l'autre. Le refus de reconnaître l'autre dans ce qu'il a de positif fixe Bruno dans un stade qu'il n'arrive pas à dépasser et engendre la possibilité du crime, du meurtre de son semblable. Bruno est considéré comme «fou », ce qui, dans le contexte de ce roman, signifie qu'il est figé dans une maladie qui réduit le rapport avec l'autre à l'alternative duelle du « lui ou moi ».

Bruno demeure, tout au long du récit, un être de chaos pulsionnel, narcissique et meurtrier. Il n'arrive pas, par la reconnaissance douloureuse de l'autre, à surmonter le déplaisir, mais se précipite dans la satisfaction de la pulsion meurtrière qui l'habite et le domine. Le meurtre est lié à son incapacité à communiquer. Somme toute, dans ce roman, il n'y a aucune possibilité de fraternité, d'échange; il ne peut y avoir que de la "frérocité », de la haine féroce du frère. Dans la confusion de l'esprit de Bruno, Étienne incarne une image négative qui éveille le négatif que Bruno porte en lui. Détruire ce négatif en l'autre en le tuant, c'est par la même occasion éteindre le négatif en soi.

Bruno s'acharne à sa " mission » $(C, 29)$ sans relâche. Il avance masqué et le piège qu'il tend à son frère est élaboré de façon méthodique, calculée, de sorte que la victime ne se rend même pas compte qu'elle est piégée. Étienne, « indifférent et sans véritable projet » $(C, 33)$, ne se doute de rien. Bruno offre à son frère des recueils du poète Chacal, qui n'est autre que son pseudonyme, afin de semer le doute et saper sa confiance. 
Bruno est revenu vivre à Sainte-Croix pour écrire sa dernière œuvre. Non pas une fiction celle-là, mais un récit de vie, le récit qu'il manipule au gré de sa vengeance. Il est revenu afin de pouvoir agir directement sur les antagonistes. [...] il est revenu pour cela, son histoire. Pour se faire justice. Et cette nuit, il a l'impression que la métamorphose obligatoire est amorcée. (C, 106-107)

Pourquoi Bruno choisit-il ce pseudonyme de chacal? Cet animal est un charognard, mais aussi un redoutable prédateur, qui est souvent accusé de tuer pour le plaisir. Dans la mythologie égyptienne, le chacal est associé à Anubis, le dieu de la mort et de l'embaumement, qui est représenté sous les traits d'un homme à tête de chacal. L'une de ses fonctions est d'accompagner les morts dans l'autre monde. Le chacal est aussi la monture de Kali, déesse hindoue du temps et de la mort, dont le nom en sanscrit signifie "celle qui détruit toute chose ». La poésie de Chacal est associée à la mort, au désespoir. Le cadeau empoisonné des recueils de Chacal participe du plan méticuleusement préparé par Bruno pour détruire son frère. Ce n'est que beaucoup plus tard qu'Étienne prendra

conscience que ses lectures de Chacal le plongeaient souvent dans une tristesse profonde, une léthargie envahissante, un dénigrement de lui-même. Bruno l'a violé jusque dans sa conscience. Même dans son inconscient. Étienne a marché à plein dans ce jeu diabolique. Il n'a rien vu, personne n'a rien vu. Il a laissé son frère le détruire jusque dans son âme. Étienne tremble de tous ses membres. $(C, 252)$

Outre le chacal, l'animal fétiche de Bruno est la chauve-souris qui, alors même qu'Étienne tente de se suicider, frôle la fenêtre de sa chambre, "revient, se pose sur le rebord, comme si elle regardait à l'intérieur » $(C$, 206). Tout comme Bruno qui ne dort jamais la nuit, ce mammifère a un mode de vie nocturne. Il est perçu comme un animal mystérieux, d'une symbolique très riche. Il est jugé particulièrement intelligent, car il sait éviter tous les obstacles quand il est en vol. Dans la culture occidentale, les chauves-souris sont assimilées au malheur ou à la mort. Elles sont souvent considérées comme la manifestation physique d'un esprit errant. Elles sont intimement associées au mythe des vampires et Bruno, avec sa fixation morbide, se nourrit du sang des vivants afin d'en tirer sa haine et sa force vitale.

Dans sa quête de destruction, Bruno évite tous les obstacles avec ruse et intelligence. II veut tout savoir, tout contrôler : " je vais pénétrer 
son silence... lui fouiller le cour... et quand le destin s'abattra sur lui, quand la vie l'aura plongé au fond de l'abîme, je serai là pour contempler ce juste retour des choses » $(C, 149)$. Bruno fouille dans le journal intime d'Étienne afin de savoir où frapper et infliger le plus de mal. II intimide Lorraine, l'amie d'Étienne, et tente de briser leur relation en révélant les incartades de son frère avec Judith. Tout est mis en œuvre afin d'agir sur son frère et le manipuler au gré de sa vengeance.

Bien qu'il ait déjà agi de façon à indisposer sa famille et qu'il inquiète les gens par son pouvoir maléfique, Bruno n'est pas soupçonné à la suite du décès de ses parents dans un accident de voiture. Lorsque l'enquête policière révèle que les freins ont été sabotés, Bruno fait porter les soupçons sur Étienne. Et puisqu'Étienne doute de tout, " de lui, de son avenir, de sa relation amoureuse » $(C, 175)$, il se défend mal lorsqu'il est accusé d'avoir provoqué la mort de ses parents afin d'hériter de la scierie.

Accusé de meurtre, déchiré entre deux femmes dont l'une porte son enfant, écrasé par les difficultés de gestion de la scierie, incertain de son avenir, de lui-même, Étienne tente de mettre fin à ses problèmes en se noyant dans la rivière. Bruno, tel Seth de la mythologie égyptienne, tente d'assassiner son frère en le noyant, mais plus rusé que Seth, Bruno réussit à pousser Étienne à accomplir son propre meurtre. Ce faisant, il termine le geste qu'il avait amorcé à la naissance d'Étienne, lorsqu'il avait symboliquement égrené des lilas, symbole de la naissance d'Étienne, et les avait regardés disparaître dans le courant de la rivière : " je sèmerai les lilas pour toi dans le courant de la rivière... et ta voix chantera éternellement dans le ruissellement de l'eau » $(C, 154)$. Bruno offre Étienne "en sacrifice au monstre de la rivière » $(C, 230)$, qui, à la différence de sa mère, ne l'a jamais trahi. Afin de profiter pleinement du spectacle, Bruno s'installe dans la berceuse de sa mère et observe de loin Étienne face au remous.

Les machinations de Bruno auraient porté, n'eût été Lorraine. Celle-ci réussit à convaincre Étienne de revenir alors qu'il est sur le point de perdre pied et d'être avalé par le tourbillon du courant. À la suite de ce suicide/ meurtre raté, des preuves supplémentaires au sujet du décès des parents sont découvertes, Étienne est disculpé du meurtre et Bruno est interné. Étienne, profondément marqué par cette succession d'événements tragiques, vend la scierie, mais perd Lorraine qui, bien qu'elle lui ait sauvé la vie, ne lui pardonne pas ses infidélités. Il tente tranquillement de refaire sa vie, tandis que Bruno continue de méditer sur les moyens de supprimer son frère.

Dans un monde sans rédemption, une rage inhumaine, une noire fureur accapare Bruno, de sorte qu'il est obnubilé par cette haine qui l'occupe tout entier. C'est une haine charnelle, ouvertement physique. Pour lui, il ne s'agit pas seulement de détruire son frère, mais de s'offrir 
une suprême jouissance sadique : celle de se baigner dans son sang. La haine est la justification de son attitude, alors qu'il invoque de grands principes poétiques. En fait, son ambition est moins d'avoir l'amour indivisible de sa mère que de jouir d'en priver l'autre et de le détruire. Le désir de conquête de l'amour de la mère est ici entièrement résorbé dans l'affirmation égocentrique du sujet, dans un amour de soi totalitaire, négateur de l'autre. Bruno devient une figure maléfique, sournoise et quasi toute-puissante, que même l'internement ne parviendra pas à mater.

Ayant survécu aux pièges de son frère, Étienne ne connaîtra jamais complètement la paix : "La nuit d'Étienne est peuplée de cauchemars. II voit la tête de Bruno partout» $(C, 304)$. Et il tente du mieux qu'il peut de recoller les morceaux de sa vie que Bruno a fait voler en éclats. Bruno, de son côté, malgré les soins hospitaliers et la médication, reste obsédé, d'où ces pages à la toute fin du roman où l'on apprend qu'il s'est procuré un objet pointu, avec lequel il a tracé "des lignes hachurées, gravées profondément dans le bois [de sa chaise berçante]. Des lettres déformées, mais parfaitement lisibles » $(C, 307)$. Bruno a tracé le nom d’Étienne, puis il l'a barré (comme il l'avait fait lors de la cérémonie de réouverture de la scierie) pour écrire par-dessus son propre nom, dans une tentative pour « effacer » le nom de son frère. Bruno a eu accès à un objet pointu que les infirmières ne trouvent plus.

Si Bruno privilégie le couteau, Étienne préfère la plume. Bien qu’il ait fait des études dans des domaines pratiques pour répondre aux attentes de son père, Étienne n'oublie pas "son rêve essentiel » $(C, 204)$, celui d'écrire de la poésie. Ce faisant, il entre en compétition avec Bruno, poète ayant déjà publié plusieurs recueils, qui juge les écrits de son frère comme des « fragments de deuil » $(C, 206)$. De plus, Étienne écrit avec une plume Mont Blanc offerte par sa mère à son dernier anniversaire. Cette plume servira à sauver la vie de Bruno lorsque, à la suite d'une crise résultant du suicide/meurtre raté d'Étienne, il gît sur le plancher, une écume blanche s'écoulant de sa bouche, en crise et hurlant : "Chacaaaaal » (C, 239). Étienne survivra à tous les complots de Bruno et, à la fin du récit, il retournera à l'écriture de la poésie, avec une plume sur laquelle Bruno a laissé la trace de sa morsure. Ce n'est que par l'écriture qu'il pourra refaire sa vie, tout en conservant les cicatrices du drame.

\section{Caïn et Abel}

Si nous revenons à la Bible, à la suite du fratricide, Caïn n'est pas condamné à mourir à son tour. Dieu ne tue pas Caïn et ne laisse pas non plus d'autres le tuer, ce qui ne ferait qu'enclencher une spirale de violence; il le marque d'un signe qui le protège, qui le rend intouchable. 
La vie humaine, même celle d'un meurtrier, est sacrée. Dieu condamne Caïn à devenir être errant et vagabond sur la terre (Gn 4 : 15-16), car le meurtre isole celui qui le commet, le coupe de sa relation avec Dieu et avec les hommes. Caïn se retire de la présence de Dieu; Bruno est interné loin du village au nom emblématique de Sainte-Croix. Il est marqué du signe de la maladie mentale et son esprit est condamné à l'errance, à la divagation. Bruno est jugé non criminellement responsable pour cause de troubles mentaux et placé sous garde dans un établissement de soins de santé mentale. Il est celui que la main de l'homme ne peut atteindre, mais il est aussi l'abominable, le maudit, isolé dans sa chambre, emprisonné dans sa folie.

Cependant, le récit de la Genèse ne se termine pas sur l'errance de Caïn. Dieu permet à Caïn de s'installer au pays de Nod. Ce pays est décrit comme étant situé à l'orient d'Éden. L'Est est le symbole de l'espérance, là où le soleil se lève. Il y a espoir d'un jour nouveau, car Caïn peut se repentir, demander pardon. De plus, sa descendance n'est pas stigmatisée, mais bâtit une ville et comptera des éleveurs, des artisans, des musiciens. Le roman de Couturier est moins miséricordieux. Bruno reste fixé dans sa haine, rigide et impénétrable, prisonnier de son projet homicide.

Abel était berger et Caïn laboureur. Par analogie, Étienne s'occupe de la brebis égarée, tandis que Bruno laboure les sillons du ressentiment. Bruno est mû par le désir d’anéantir la vulnérabilité d’Étienne dans laquelle il ne se reconnaît pas, mais Étienne au contraire éprouve le devoir de protéger celle de Bruno. Étienne accepte sa responsabilité d'assumer la faiblesse, la fragilité et la vulnérabilité de son frère. Il est responsable d'autrui, sans attendre la réciproque, au risque même de perdre la vie. "Sa seule famille, maintenant, dira-t-il au psychiatre soignant Bruno, c'est moi » $(C, 246)$.

Après le meurtre d’Abel, le Seigneur interrogea Caïn, qui lui répondit avec arrogance : "Suis-je le gardien de mon frère? »(Gn 4 : 9) Si Bruno récuse toute affiliation avec Étienne, ce dernier continue d'aider son frère; il assume sa responsabilité et devient le gardien de son frère. Étienne est le nom du protomartyr, le premier martyr de la foi chrétienne, celui qui est lapidé pour avoir voulu convertir et secourir ses frères juifs. Dans le contexte du roman, la réplique de Caïn s'applique à Étienne, qui, en toute connaissance de cause, accepte la responsabilité, l'exigence éthique d'être le gardien de son frère. Toutefois, Étienne est un personnage faible, qui ne semble pas mesurer la haine de son frère, tout comme Abel, que nous ne connaissons qu'à titre d'innocent assassiné.

Si Bruno est un être violent qui se définit en fonction d'Étienne, qu'il doit détruire, Étienne se définit en fonction de son père, qu'il admire et pour qui il ressent une sorte d'émulation. Étienne cherche à profiter de 
l'exemple que lui donne son père et à se montrer à la hauteur de la tâche qu'il lui léguera. Tout comme Adam, le père d'Abel, Georges Bellefleur s'est construit un jardin "pour le cultiver et le garder » (Gn 2 : 15). Le père d'Étienne est l'un de ces hommes plus grands que nature, qui a réussi à asservir la forêt, à l'exploiter afin qu'elle livre ses richesses à la scierie dont il est le fondateur et l'unique propriétaire. Il a connu l'époque des camps de bûcherons en hiver, de la drave au printemps, puis celle de la mécanisation et de la mondialisation de l'industrie. Face à un tel démiurge, Étienne pourrait redire les vers d'Alfred Desrochers :

Je suis un fils déchu de race surhumaine, Race de violents, de forts, de hasardeux [...]

Par nos ans sans vigueur, je suis comme le hêtre

Dont la sève a tari sans qu'il soit dépouillé,

Et c'est de désirs morts que je suis enfeuillé

Quand je rêve d'aller comme allait mon ancêtre ${ }^{7}$

Étienne n'a ni la stature, ni le dynamisme, ni la force physique et morale de son père. Âgé de presque trente ans, il est encore traité comme un enfant et ne sait pas ce qu'il veut. Il croit que sa « main n'aura jamais la force de celle de son père » $(C, 101)$ et ses doigts effilés ne peuvent même pas retenir l'alliance de son père, qui « tombe sur le bureau » $(C, 277)$. Étienne " n'a pas [l]a carrure » $(C, 277)$ de son père. Georges Bellefleur, que son épouse surnommait le "bulldozer» $(C, 15)$, qui n'a même pas terminé un cours secondaire et qui pourtant a mené à bien toutes ses entreprises. Il a fondé et géré une scierie qui fait vivre tout le village, qui "nourrit à peu près toutes les familles de la région" $(C, 36)$. Son fils, malgré ses diplômes en administration des affaires et en droit, se révèle faible et indécis, incapable de prendre la relève.

Étienne vendra l'héritage à un anglophone du nom de Blackster. Cette étoile noire ou ce soleil noir ne renvoie-t-il pas au "Soleil noir de la Mélancolie » du poème «El desdichado » de Nerval, dont le titre signifie le « malchanceux », le « malheureux », et, pourquoi pas, le " déshérité »? Étienne est effectivement le malheureux, le déshérité qui traîne son désespoir et son sentiment d'inadéquation du village de Sainte-Croix à New York (où, à partir du 11-Septembre, "son propre destin s'est précipité » $[C, 282]$ ), à Athènes et aux pyramides d'Égypte (où auraient dû se rendre ses parents, s'il n'y avait pas eu l'accident de voiture), sans réussir à trouver sa voie, sans réussir à regagner l'amour de Lorraine, la mère de sa fille.

7. Alfred Desrochers, "Je suis un fils déchu », À l'ombre de l'Orford, Montréal, Fides, coll. « Nénuphar», 1948, p. 35-36. 
Toutefois, Étienne conservera une partie de son héritage paternel, un "paradis » $(C, 147)$, une " terre intacte » $(C, 148)$ où certains arbres sont plus que centenaires. Dans cette " petite parcelle de bonheur » $(C$, 148) où le père a avoué que le fils avait été conçu, Étienne se construit un pavillon de bois rond et surnomme cette alcôve le Bois des songes. Dans sa solitude, Étienne tente de se mettre sérieusement à l'écriture et de se guérir : "Ne demander à la vie que ce qu'elle peut nous offrir. Le reste, se l'inventer soi-même » $(C, 307)$. Protégé de la rivière par une falaise (et de Bruno par les murs de l'hôpital psychiatrique), Étienne devine la présence de celle-ci «sous sa couche de glace, qui file inlassablement vers la mer... » $(C, 308)$, car toute menace n'est pas définitivement écartée.

$$
\star \star \star
$$

Dans Chacal, mon frère, Gracia Couturier réinterprète le grand récit des frères ennemis. Son roman d'une tentative de fratricide raconte l'universalité du mal et son caractère mystérieux et implacable. L'épisode de Bruno et d'Étienne n'est pas une simple « histoire de famille », mais s'inscrit dans une tragique lignée, qui tire son origine des premiers récits de l'humanité. Tout dans le destin des deux frères accuse leurs différences. À l'énergie pulsionnelle de Bruno s'oppose la fragilité d’Étienne. À la vitalité quasi barbare et cruelle de l'aîné s'oppose l'excès de sensibilité douloureuse et d'intellectualité du cadet. Toutes ces particularités qui singularisent les deux frères forment et renforcent deux pôles contradictoires et opposés dans un huis clos de déchirure et de destruction tel que nous n'en trouvons que rarement en littérature acadienne.

La question est maintenant de savoir comment l'auteure, qui travaille sur une suite à son roman, pourra maintenir cette tension et cette représentation du mal à ce degré de perfection. Certes, Bruno est puni et interné, de sorte que le venin qu'il sécrète le mine et le torture, mais il ne peut être retenu. Il a accès à un objet pointu que les infirmières ne retrouvent plus et il tuera son frère ou ils s'entretueront, mais aucune autre solution n'est envisageable face à cette force du Mal. Dans l'eschatologie nordique, le Ragnarök, il est prophétisé qu'une grande bataille aura lieu entre les forces du Mal et celles du Bien. Le dieu Thor se battra contre le serpent de Midgard et le tuera, mais il ne fera que neuf pas avant de tomber mort à son tour, en raison du venin que le serpent aura craché sur lui. Que Bruno soit interné ou tué, il a instillé son venin dans Étienne, qui se promène de par le monde, reprenant le trajet que voulaient emprunter ses parents, visitant des ruines, déjà à demi mort. Dans cet univers romanesque sans miséricorde, la lutte entre le Bien et le Mal, la Lumière et les Ténèbres, l'esprit saint et l'esprit mauvais est une lutte sans fin, qui ne peut mener qu'à la mort. 